näherte, nahm or eine duarakteristisdie "Lanerstellung" ein, d. h., er hob den Körper vom Boden hoch, indem die langen Beine näher an den Körper herangezogen wurden. Außerdem wird ein Bein des 2. langen Tastbeinpaares leicht angehoben. Nähert sich nun dic Flicge, so läßrt sic der Weberknedht so nahe an sidh herankommen, dals er sie mit einem Satz erreichen kann. Bcim Beutefang treten Cheliceren, Palpen und 2. Beinpaar in Tätigkeit. Die Cheliceren werden in die Weichteile drr Beute geschlagen, während Palpen und 2. Beinpaar sic unklammern. Das Opfer wehrt sich meist verzweifelt und versudit zu cntkommen. Platybunus häl die Beute so lange umklammert, bis sie zu ermatten heginnt. Der Weberknedht scheint keine Gif tdrüsen zu besitzen, denn die gefangenen Tiere wehren sich of nod sehr lange und sind keineswegr gr. lähmt. Selbst wenn Platybunus beginnt, die Beute zu verathren, hewegt sidh diese noch; er frikt sie also "bri lehendigem Leibe".

Audh im Freien wurde Platybunus bucephalus heim Verzehren cines - allerdings nidit mehr bestimmbaren Dipters angetroffen. Eine besonders aufrällige Erscheinung ist die außerordentlich starke Neigung zum Kannibalismus bei Platybunus bucephalus. Läßt man mehrere Tiere zusammen in eincm Zuchtgefäß, so dauert es nur $1-2$ Tage, bis sich die Weberknechte gegenseitig getötet und gefressen haben, so daß schliellich nur nod das größic und kräftigste Exemplar übrigbleibt. Dicser Vorgang ist nicht otwa mit Nahrungsmangel m erklären, denn in den ZuditgefäBen hefanden sich bei den Versuchen stets genügend Fliegen. Selbst in der Natur wurden hiufig Platybumus angetroffen, die gerade dabei waren, einen Artgenossen mu verspeisen. Weiterhin jst interessant, daß nidht kopulationswillige Weibden von Platybunus bucephalus, die die Mänuchen an Körpergröße beträchtlich ähertreffen, diese angreifen und oft sogar töten und verzehren, wenn sie sich ihnen nähern. Ahnliche Vorgänge wurden bisher nur bei Spinnen beobaditet.

Schon dieser kurze $A b r i \beta$ zeigt, daß die Ernährung der räuherischen Weberknechte eine schr vielfältige ist. Allerdings sind unsere Kenntnisse auf diesem Gebiet noch immer dïftig und es gäbe hestimmt noch viele interessante Probleme zu lösen.

\section{Lileraturverzeidnis}

Fo r hacdt: Nods Kästuer (1928); Opiliones. Die Tirrwell Deutsehlandk, Lieferung 8 , Jenn.

R ii $\mathrm{h}$ : Nath Kustuer (1928): Opiliones. lbid

3. C. L. Koch: Nadi Kästaer (1928): Opiliones. Ibid.

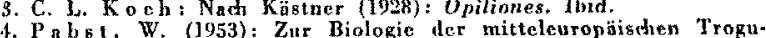
Pabst, W. (1953): Zur Biologie der mitteleuropäis

5. I m m r r V V. (1954): Zur Bialogie nnd Physiologie von Nemastoma I morl, V. (1954): Zur Bialogie und Physiologie ron Nemastoma
guadripunctatum (Opiliones, Dyspnoi). Zool. Jb., Abrtg. f. Syste-

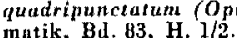

6. Aimsky.Korsakow (1924): Die Kugelhaare von Nemastoma lagubre. Zool. Anz., Leipzig 1924. Bd. 60 .

7. I m mel, V. (1955): Einige Bermerk ungen zar Biologin van Platy. bunus bucephalus (Opiliones, Eupnoi). Zool. Jh., Abtlg. f. Systematik. Im Brudk.

\title{
Die Ähnlichkeit der Larven von Lilienhähnchen und Kartofielkäfer
}

\author{
Von H. Ḧ̈RDTL
}

Während eines Kartoffelkäfersudhdienstes im Juni wurden mehrere rote Larven abgegehen, die bei fü̉htiger Betrachtung leicht als Larven des Kartoffelkäfers angesprochen werden konnten. Die Bestimmung ergab: Larven des Lilienhähnchens, Crioceris merdígera $\mathbf{F}$. Einige Hinweise auf Besonderheiten der Larve gegeben, diirften angebracht sein als Ergänzungen der im Schriftum vorliegenden Angaben.

REINECK schreibt über dic Larve des Lilienhähnchens folgendes: "Die erwachsenen Larven haben 12 Segmente, die nach hinten zu mehr oder weniger blasig aufgetrieben sind. Sie sind $7-8 \mathrm{~mm}$ lang, hell fleisdhrot. Der Kopf, die Oberseite des 1. Ringes und die 3 Beinpare sind sehwärzlich. Die Fühler sind schr kurz, dreigliedrig. Der 3. und 4. Leibesring hat seitlich je 2 größere Tuberkeln, der 4 . bis 12. Ring besitgt je eine kroisrunde, angedunkelte Stigmenöffnung. Die Larven gehen zur Verpuppung in die Erde." Eine vergleichende Aufstellung der Merkmale bringt auch GersDorf.

Die meist vorhandenen Kotmassen, dic die Larven einhüllen, fehlten. Ansonsten scheint diese Kothülle recht harakteristisch zu sein. Die Larve ist aber für Bestimmungszwecke in den Nachschlagebüchern unklar beschrieben. So bezeichnet PAPE dic Larve schmutzig grau bis rötlich, mit schwarzen schleimigen Kotmassen bedeckt. SCHRöDER bezeichnet sie als orangegelb his gelblich weiß. Bei Flachs wird die Kothülle hervorgehoben, nicht aber Farbe und Form der Larve.
Wie bei der Kartoffelkäferlarve, so finden wir auch beim Lilienhähnchen die blasige Auftreibung, desgleichen bei der gelblichen Larve des Getreidehähndiens (APPEL) und der graugelben Larve des Zwiebelhähnchens (Котте). Es fehlen die für die Kartoffelkäferlarve charakteristischen seitwärts liegenden schwarzen Pigmentflecke an den Leibesringen.

Als Futterpflanze dient der Larve des Lilienhähnchens vor allem Lilienarten. Auf Kartoffelkraut scheint diese Larve nur selten vorzukommen (vgl. Hesse). Eine gewisse Ahnlichkeit beider Larven ist gegeben. Thre Kenninis ist angezeigt, um gegebenenfalls die durch einen falsch gemeldeten Fund entstehenden Folgen auszuschalten, wie es die Meldung eines neuentdeckten Kartoffelkäferherdes mit sich bringt.

\section{Literaturverzeichnis}

Appel, o.: Tasdicnatla der Getreidekrankheiten. Berlin 1931. Alachs, K.: Krankheiten vml. Parakiten der Zierphanzen. Stutgart 1931

Gers d orf, Er.: Anzeig. f. Sdädlingskle. 14, 42-43, 1938. Hesse, Er: Ztedir. f. wise. Insektegbiologie, 27, 85-87, 1934 . K I c in o, R.: Entomal. Blätter, 32, 84, 1937.

Kot te, W.: Krankheiten und Sdididinge im Gemüsebau und ihre Bekämpfung. Berlin 1944 .

$P$ a p c, H.: Dic. Prnxis der Bekämpfung ron Krankheiten und Sctüdlina pen der Zierpflanen. Berlin 1939 .

Reli, L.: Jnhrb. d. Hamburg. wissensd. Anstalt., 19, 113-223, 1909.

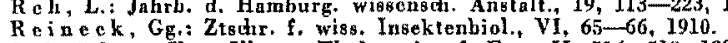

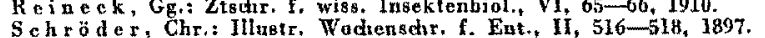
Tulgrea, A.i Uppsater i praktikk Euton., Uppsala 22, 43-134, 1913.

\section{Kleine Mitteilungen}

\section{Mitteilungen}

Internationale Pflanzenschubsizung

Im Anschluß an die diesjährige $W$ anderausstellung der DLG in München fand am 17. Mai d. J, unter dem Vorsizs von Ministerialrat Dr. Drees eine internationale Pflanzenschubsitzung statt, an der nelien dem Bayerisden Staatsminister für Eraihrung, Landwirtschaft und Forsten,
Herr Prof. Dr. B a un ga 1 u e r, der Generaldirektor der EPPO, Herr Dr. Wilkin s (Paris), der Herr Präsident der BBA, Vertreter der Nacbbarländer, der Vorsigende des Arbeitskreises der $D_{t}$. Pflanzenschutgmittelindustrie und die Vorstände der Pfanzenschuzämter der Länder teil. uahmen. Neben Fragen der Pflanzenschuz-Quarantäne bei Ein- und Ausfuhren stand ein anregender Vortrag von 